\title{
EL INTERÉS DE LOS LATINOAMERICANOS POR LOS ASUNTOS INTERNACIONALES
}

\author{
The Interest of Latin Americans in International Affairs \\ O Interesse dos Latino-americanos nos Assuntos Internacionais
}

\section{MIGUEL ÁNGEL LÓPEZ VARAS @i] mlopez@uchile.cl 1}

\author{
1 Universidad de Chile \\ Envío: 2020-04-21 \\ Aceptado: 2021-03-23 \\ First View: 2021-04-27 \\ Publicación: 2021-05-31
}

\begin{abstract}
Palabras clave: interés político; conocimiento político; noticias internacionales; relaciones internacionales; medios de comunicación

\section{Resumen}

Si bien existen numerosas investigaciones sobre el interés y el conocimiento de los ciudadanos sobre los asuntos internacionales en los Estados Unidos y Europa, los estudios comparados sobre estos temas en América Latina siguen siendo escasos. Este artículo pretende extender el conocimiento sobre este fenómeno a la región usando la encuesta "Las Américas y el Mundo" realizada en 2014/5 en siete de los principales países de América Latina. Si bien existen diferencias entre países, este artículo busca identificar patrones comunes como el amplio interés que tienen los latinoamericanos por las noticias internacionales, especialmente cuando estas implican a su país de origen. Sin embargo, los resultados preliminares cuestionan la existencia de una brecha de género en el consumo de noticias internacionales, identificada por la literatura comparada.
\end{abstract}

Keywords: political interest; political knowledge; international news; foreign affairs; mass media

\section{Abstract}

While there is extensive research on citizens' interest and knowledge on international affairs in the United States and Europe, comparative studies on these issues remain scarce in Latin America. This paper aims to extend knowledge about this phenomenon in the region by using the survey "The Americas and the World", conducted in 2014/5 in seven of the main countries in Latin America. Although there are differences between countries, this paper seeks to identify common patterns, such as Latin Americans' wide interest in international news, especially, when they related to their country of origin. However, these preliminary results call into question the existence of a gender gap in the consumption of international news, identified by comparative literature. 
Palavras-chave: interesse político; conhecimento político; notícias internacionais; relações internacionais; meios de comunicação
Enquanto existem numerosas pesquisas sobre o interesse dos cidadãos nos assuntos internacionais nos Estados Unidos e na Europa, os estudos comparativos sobre esse tema na América Latina continuam escassos. Este artigo tem como objetivo ampliar o conhecimento sobre esse fenômeno para a região, utilizando a pesquisa "As Américas e o Mundo" realizada em 2014/5 em sete dos principais países da América Latina. Embora existam diferenças entre os países, este artigo procura identificar padrões comuns, como o interesse dos latino-americanos pelas notícias internacionais, especialmente quando envolvem seu país de origem. No entanto, esses resultados preliminares questionam a existência de uma lacuna de gênero no consumo de notícias internacionais, identificada pela literatura comparativa.

\section{INTRODUCCIÓN}

En la medida que el mundo se vuelve cada vez más globalizado, las distancias entre las naciones se van reduciendo y muchos problemas nacionales tienen ahora tintes regionales o globales lo que aumenta el interés de la ciudadanía por los asuntos globales. Las noticias del exterior son hoy más relevantes que en el pasado debido a la cobertura de eventos y asuntos internacionales de forma instantánea sin importar la lejanía geográfica. Vivimos en un mundo de comunicación en tiempo real, facilitado por la emergencia de nuevas tecnologías como el internet, los teléfonos celulares, la comunicación satelital, los canales de noticias de 24 horas, y redes sociales como Twitter, Facebook, YouTube e Instagram. Esta revolución comunicacional disparada por los nuevos medios, las redes sociales y las adaptaciones de los antiguos medios al internet no solo han propiciado la presencia de un público más atento e interesado con lo internacional sino que además "han cambiado de manera fundamental e irreversible el significado del poder en las relaciones internacionales, la elaboración de las políticas de defensa y relaciones exteriores, y la conducción de la diplomacia" (Gilboa, 2002, p. 731).

Este artículo, basada en los datos de la encuesta "Las Américas y el Mundo", trata de mostrar en perspectiva comparada los grados de interés que tiene la ciudadanía de América Latina con los asuntos internacionales, el uso de los medios de comunicación masiva para informarse de los sucesos mundiales y el grado de conocimiento de asuntos internacionales.

El principal objetivo es someter a comprobación en América Latina tres supuestos que emergen de la literatura comparada. El primero señala que las noticias internacionales importan, aunque menos que las noticias nacionales. En segundo lugar, que existe una brecha de género en el consumo de noticias internaciones, dado su menor interés en estos asuntos. Y finalmente que el alto interés declarado no implica conocimiento de la actualidad internacional. 


\section{OPINIÓN PÚBLICA Y NOTICIAS INTERNACIONALES}

La literatura comparada, basada en investigaciones para el caso de Estados Unidos consideró por muchos años que la información y noticias internacionales no concitaban la atención de la ciudadanía en los países desarrollados. Esta creencia, conocida como la "mood theory" o el llamado "consenso de AlmondLippmann" mantenía que la opinión pública sobre asuntos internacionales era volátil, carecía de coherencia y estructura y que en definitiva tenía poco impacto en política exterior (Almond, 1960, p. 53; Holsti, 1992). Se pensaba que la indiferencia era la respuesta típica de la ciudadanía a preguntas de conocimiento o interés sobre política exterior. En momentos de crisis internacionales o la posibilidad de una guerra esa indiferencia se podía transformar en una aprehensión vaga, incluso algo de fatalismo e ira, pero siempre sólo representaba un estado de ánimo "temporal y caprichoso" (Klingberg, 1952, p. 240). Sin embargo, el alto interés del público norteamericano por la guerra de Vietnam rompió este consenso y hoy la literatura comparada reconoce que las noticias internacionales importan en la opinión pública, que ésta es estable, y que posee una estructura latente reconocible (Caspary, 1970; Holsti, 1992; Olmastroni, 2017).

Desde un punto de vista teórico el consenso de Almond y Lippman responde a un período dominado por el enfoque realista de las relaciones internacionales. Para Hans Morgenthau (1948) y los realistas el interés nacional era prioritario en las relaciones internaciones y trascendía los estados de ánimo y las pasiones de una opinión pública desinformada e ignorante (Holsti, 1992; 2004; Morales y Shiavon, 2015; Drezner, 2008). En cambio, la valoración de la opinión ciudadana en asuntos internacionales se remonta a Kant y Bentham y a la tradición democrático-liberal que considera que la rendición de cuentas de los gobernantes ante la ciudadanía "puede frenar las inclinaciones de los líderes a la guerra" (Holsti, 1992, p. 440). Para los liberales la relación entre la opinión pública y la política exterior es deseable ya que la primera es fuente de legitimación de la segunda, y en democracia la política exterior no debería diferenciarse del resto de las políticas públicas en el escrutinio ciudadano (Morales y Shiavon, 2015). Para otros esta visión contrapuesta de la importancia de la opinión pública entre realistas y liberales no es más que la expresión de elitistas contra pluralistas (Smith, Hadfield y Dunne, 2016, pp. 188-90).

En la literatura se han identificado diversos factores que influyen en el interés y conocimiento de lo internacional. Esos se pueden agrupar en factores contextuales o sistémicos relacionados con el output noticioso de los medios hacia la ciudadanía y otros de naturaleza individual referidos a las variaciones en interés según género, edad, educación, etc. (Bennett et al., 1996; Mouron, Hardt y Onuki, 2018). 


\section{a. Factores contextuales}

La gran mayoría de los latinoamericanos tiene limitada experiencia personal en otros países o continentes diferentes al suyo ${ }^{1}$. Para conocer el mundo y mantenernos informados de sus cambios y transformaciones dependemos, entonces, de las representaciones proporcionadas por los diversos medios de información audiovisual, escritos o electrónicos. Esto hace que el interés esté influido por el tipo de oferta de los medios de comunicación en materia internacional (Jerit, Barabas y Bolsen, 2006; Smith, Hadfield y Dunne, 2016, pp. 190-196).

Numerosos autores han identificado diversos factores que predisponen que determinados asuntos domésticos o regionales se conviertan en noticias internacionales. Entre ellos están las noticias referentes a las naciones más poderosas, los países con los existe proximidad cultural o geográfica, las naciones con las que se poseen vínculos históricos, económicos o políticos o cuando el asunto internacional afecta el interés nacional del país receptor (Galtung y Ruge, 1965; Lee y Yang, 1996; Wu, 2000). Otros han destacado que los países ubicados en el centro del sistema mundial aparecen con frecuencia en los medios, mientras que los de periferia solo adquieren importancia noticiosa en tiempos de crisis, desastres naturales y guerras (Chang, 1998; Joye, 2010).

Otro factor contextual tiene que ver con el papel de "gatekeepers" de los medios locales. Ellos realizan este trabajo profesional influenciados por intereses personales o del medio, las líneas editoriales, las rutinas, posiciones ideológicas y aspectos culturales (Galtung y Ruge, 1965; Nossek, 2004; Grasland, 2020).

Finalmente, un factor contextual indirecto, y poco tratado en la literatura, tiene que ver con el grado de apertura y transparencia del proceso de toma de decisiones de los estados en política exterior, y de cuánto de eso es recogido por los medios (Steiner, 1987; Naveh, 2002). De esta forma, procesos de toma de decisiones más abiertos como en Uruguay (Garcé et al., 2018) tendrían más incentivos para incorporar a la opinión pública y recurrir a la prensa que procesos más cerrados como los que se dan en Chile (Garcé et al., 2018) o en Brasil (Lopes, 2013).

1 Según la encuesta Las Américas y el Mundo (2014) en promedio el 73 \% de argentinos, brasileños, chilenos, mexicanos, peruanos, colombianos y ecuatorianos declara que nunca ha salido de su país. Los argentinos son lo más cosmopolitas ya que $26,4 \%$ o de ellos manifiesta que ha viajado fuera tres veces o más y $19,51 \%$ reconoce haber salido del país una o dos veces. En segundo lugar se ubican los chilenos donde el $17,31 \%$ reconoce haber viajado una o dos veces y $16,19 \%$ tres o más veces y más atrás los colombianos. Los menos cosmopolitas son los brasileños pues el $87 \%$ de ellos señala no haber viajado al exterior. 


\section{b. Factores a nivel individual}

Gran parte de la literatura comparada señala que existe una brecha de género en el consumo de noticias, ya los hombres tendrían más interés por la política que las mujeres (Norris, 1997; Poindexter et al., 2008; Benesch, 2012; Newman et al., 2013; Pew Research Center, 2007; 2018). Incluso algunos han señalado que en EE. UU. (Pew Research Center, 2007) y Europa (Reuters, 2014, p. 50) los varones preferirían las noticias políticas nacionales e internacionales y las relativas a deportes, mientras las mujeres se interesarían más por información vinculada a estilo de vida y entretenimiento. Sin embargo, las mujeres serían más proclives que los hombres a descubrir noticias nuevas en las redes sociales y a compartirlas por diversas plataformas digitales (American Press Institute, 2014).

Otro variable a nivel individual que tendría importancia en el interés por lo internacional es la edad. En algunos países como el Reino Unido se ha descubierto que el consumo de noticia internacionales aumentaría en la medida que aumenta la edad de los ciudadanos (Levy, 2012), pero en otros como EE. UU. el máximo interés se concentraría en los rangos de 30 a 39 años y en los mayores de 60 años (American Press Institute, 2014).

Otras variables comúnmente usadas para explicar interés y sobre todo conocimiento de noticias internacionales son educación y variables socio-económicas como ingresos, niveles socioeconómico o GDP per cápita (Bennett et al., 1996; Mondak, 2001; Mouron, Hardt y Onuki, 2018; Batista, 2020).

\section{EL INTERÉS LATINOAMERICANO POR LOS ASUNTOS INTERNACIONALES}

Este análisis está basado en los datos de la encuesta Las América y el Mundo 2014 realizada en Argentina, Brasil, Chile, Colombia, Ecuador, México y Perú. Esta encuesta fue levantada a fines del 2014 y principios de 2015 de manera presencial en hogares rurales y urbanos y, a diferencia de los levantamientos anteriores y posteriores, posee una completa batería de preguntas de interés y conocimiento internacional ${ }^{2}$. Lamentablemente no existe una base de datos unificada que incluya todos los países y algunas de las preguntas de caracterización de los entrevistados no usan escalas similares o que se puedan homogeneizar. Entre ellas están algunas variables muy importantes para explicar consumo de noticias y conocimiento de asuntos internacionales como educación, ingresos o nivel socioeconómico.

2 La información metodológica de los levantamientos de la encuesta está disponible en https://www. lasamericasyelmundo.cide.edu/ 


\section{Gráfico 1. Interés en Asuntos Nacionales e Internacionales (Mucho y Algo interés)}

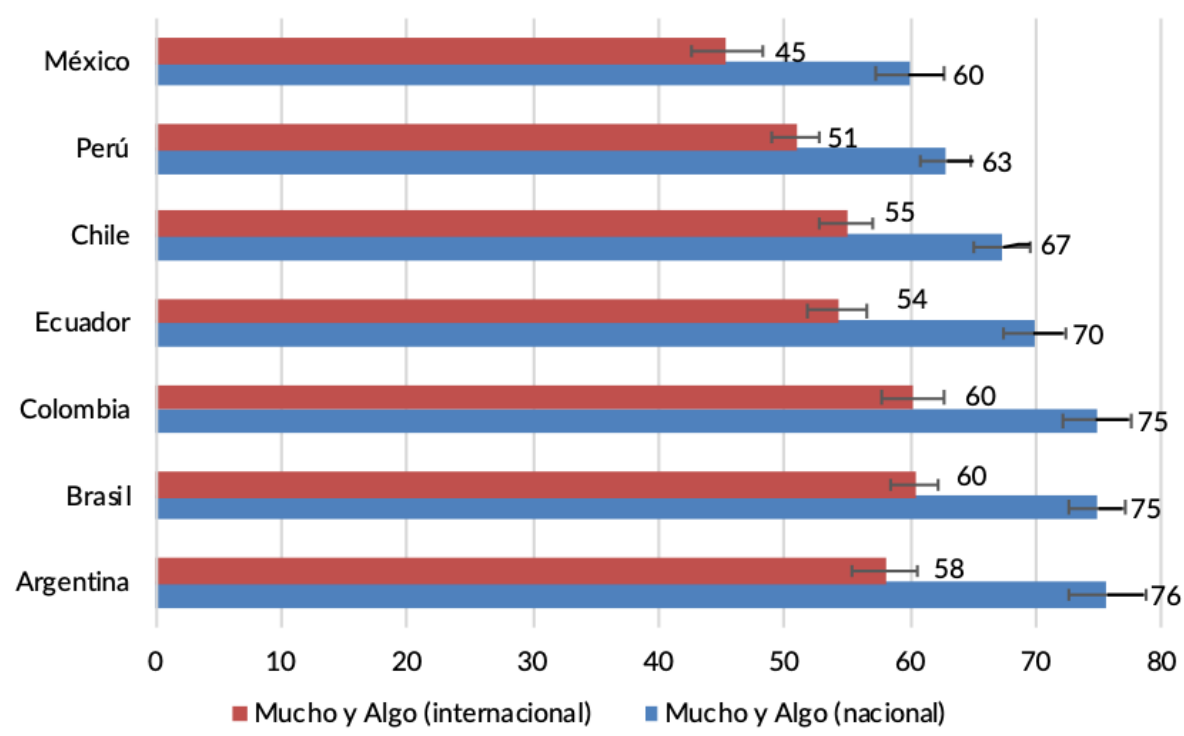

Fuente: Las Américas y el Mundo 2014.

El gráfico 1 muestra el interés de los latinoamericanos por los asuntos nacionales e internacionales en perspectiva comparada. Al igual que en los países desarrollados (Reuters, 2014, p. 50) o en estudios a nivel global (Aalberg et al., 2013; Pew Research Center, 2018) las noticias nacionales atraen más la atención por razones de cercanía, sin embargo en todos los países, con la excepción de México, los asuntos internacionales importan mucho o algo a más del $50 \%$ de la población. Llama la atención los casos de Colombia, Brasil y Argentina donde el interés por lo internacional bordea el $60 \%$, aunque en estos países el atractivo por lo nacional también es mayor, por lo que puede tratarse de países con una ciudadanía más informada. De cualquier forma, no se puede descartar que el alto interés por las noticias nacionales e internacionales sea efecto de un sesgo de deseabilidad so$\mathrm{cial}^{3}$. Este sesgo también podría existir cuando se pregunta a la ciudadanía por su interés en las noticias internacionales cuando involucra directamente a su país. En ese caso el interés sube en promedio 15 puntos porcentuales en todos los países excepto en Brasil (sube 8,5) y especialmente en México (sube 4,8).

3 El sesgo de deseabilidad social es la tendencia de los individuos a presentarse de la manera más favorable en relación con las normas y costumbres sociales predominantes cuando responde un cuestionario (Crowne y Marlowe, 1960; Lavrakas, 2008). 


\section{Gráfico 2. Interés en Asuntos Nacionales e Internacionales por sexo (Mucho y Algo interés)}

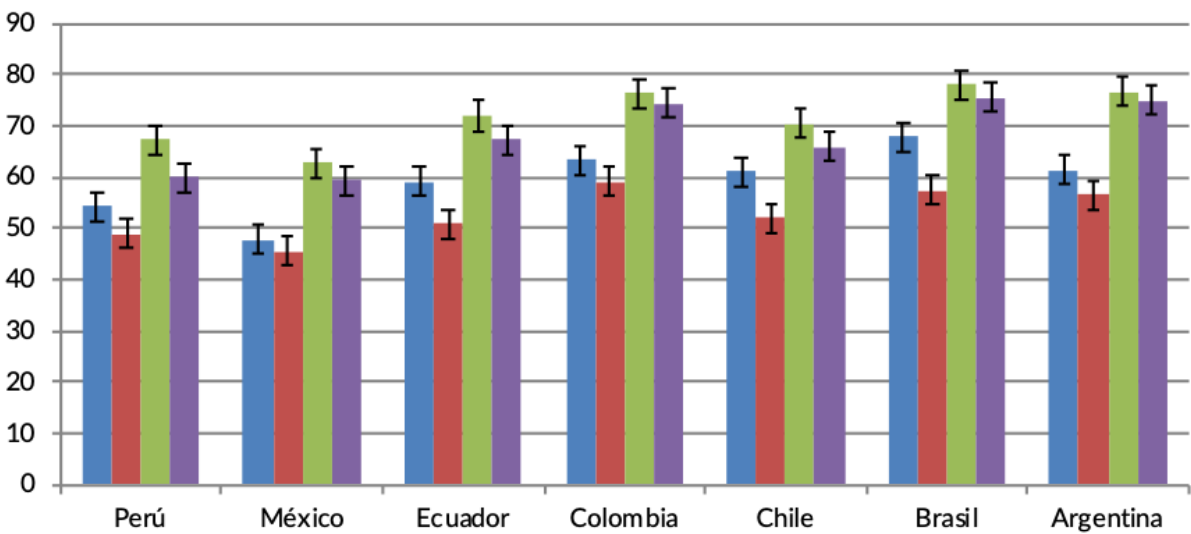

- Internacional Hombre $\quad$ Internacional Mujer Nacional Hombre $\quad$ Nacional Mujer

Fuente: Las Américas y el Mundo 2014.

A diferencia de lo señalado por la literatura comparada, nuestro análisis del interés por los asuntos nacionales e internacionales por sexo (Gráfico 2) encuentra diferencias significativas únicamente en algunos países. La estimación de los intervalos de confianza y las pruebas de chi2 solo muestran una diferencia con respecto a esta variable en los casos de Ecuador, Chile y Brasil, pero esta brecha de género en el interés por los asuntos internacionales es insignificante ( $V$ de Cramer cercana a 0,1 ). Algo similar ocurre si comparamos las medias (prueba T para muestras independientes), donde dicha brecha se confirma nuevamente solo para esos tres países ${ }^{4}$. Esta discrepancia con la literatura comparada puede ser efecto de las diferencias en el "question wording", ya que la encuesta Las Américas y el Mundo usa una pregunta más amplia que refleja el interés general por lo internacional, donde podría existir nuevamente un sesgo de deseabilidad social. En cambio, la mayoría de los estudios comparados que apoyan la existencia de una brecha de género se han centrado de manera más específica en el consumo (consumption) de noticias internacionales.

Si controlamos la variable por grupos de edad en el conjunto de países ${ }^{5}$ no existen diferencias significativas entre edad y el interés por lo internacional (Gamma 0,07). Tampoco existe una relación al repetir el análisis por país, sin embargo,

4 Esto no es recomendable, pues consideramos la variable interés, que es ordinal, como una variable numérica.

5 Los análisis de rangos etarios no consideran a Brasil y Argentina pues no se dispone de la información. 
los jóvenes (de 18 a 25 años) mexicanos y colombianos estarían menos interesados en los asuntos internacional que los jóvenes de los otros países.

Interés no implica conocimiento de los asuntos internacionales. Las mediciones de conocimiento son útiles para controlar el sesgo de deseabilidad social y para conocer qué tan informados están los ciudadanos de los asuntos internacionales, sobre todo si reconocemos, como la tradición liberal, que los públicos importan en política exterior (Bennett et al., 1996).

Tabla 1. Conocimiento de siglas (Sólo respuestas correctas)

\begin{tabular}{lccccc}
\hline & ONU & OEA & FIFA & Moneda UE & Capital China \\
\hline Brasil & 31 & 9 & 18 & $\mathrm{n} / \mathrm{a}$ & $\mathrm{n} / \mathrm{a}$ \\
Chile & 64 & 46 & 70 & 54 & 27 \\
Colombia & 62 & 57 & 65 & 57 & 29 \\
Ecuador & 55 & 46 & $\mathrm{n} / \mathrm{a}$ & 49 & 13 \\
México & 52 & 16 & 37 & 40 & 23 \\
Perú & 60 & 52 & 47 & 47 & 22 \\
Argentina & $\mathrm{n} / \mathrm{a}$ & $\mathrm{n} / \mathrm{a}$ & $\mathrm{n} / \mathrm{a}$ & 74 & 35 \\
\hline & & $\mathrm{n} / \mathrm{a}=$ sin información. & &
\end{tabular}

Fuente: Las Américas y el Mundo 2014.

La tabla 1 muestra que, a pesar de en los países estudiados los ciudadanos reconocen un gran interés por los asuntos internacionales, sus niveles de conocimiento varían por país cuando se les pide identificar siglas de organismos internacionales. Por ejemplo la ONU, el organismo supranacional más importante del mundo, fue ampliamente reconocido en Chile, Colombia y Ecuador. Una cosa similar ocurrió con la Organización de Estados Americanos (OEA) y con la Federación Internacional de Fútbol Asociación (FIFA). En los tres casos se demuestra que son los ciudadanos de Chile, Colombia y Perú los que tienen menores problemas para identificar siglas internacionales y Brasil y México los que más desconocen estos organismos. En el caso mexicano esto no es sorprendente, pues, como señalamos anteriormente, solo un $45 \%$ manifiesta un interés por los asuntos internacionales. Llama la atención el caso de Brasil, donde al igual que Colombia un 60 \% de las personas reconoce interés por lo internacional, pero su nivel de conocimiento está muy por debajo de la media de los otros países. Incluso en una nación como la brasileña en donde el fútbol está muy presente, solo un $18 \%$ identificó a la FIFA, muy por debajo de Chile (70 \%) e incluso de México (37\%). Algo similar ocurrió 
cuando se les pidió nombrar la Moneda de la Unión Europea y la capital de China. En estos casos, los argentinos demostraron tener más conocimientos, seguidos por colombianos y chilenos.

La metodología más usada para medir conocimientos considera una escala común por países que examine las preguntas correctas a través de un índice (Mondak, 2001; Elff, 2009; Batista, 2020). Desafortunadamente esto no es posible, pues como muestra la Tabla 1, muchas preguntas de conocimientos fueron omitidas en varias encuestas nacionales, por lo que algunos autores se han podido centrar en el estudio de un caso como Brasil al utilizar estos datos (Mouron, Hardt y Onuki, 2018). Sin embargo, un estudio reciente ha demostrado que la medición de los niveles de conocimiento es muy dependiente del tipo de escala utilizada (Batista, 2020).

Al analizar cuáles son los principales medios de comunicación por lo que se informan los latinoamericanos en general, la televisión se impone por lejos. En promedio el $86 \%$ de los ciudadanos de los países estudiados reconocen que siguen las noticias por televisión todos los días o al menos algunas veces por semana. Esto no cambia al considerar el sexo de los encuestados, donde los porcentajes son muy similares, al igual que por rango de edad. Aunque comparativamente los jóvenes (de 18 a 25 años) en general ven menos televisión que sus mayores, en promedio $84 \%$ de ellos sigue prefiriendo a la televisión como su principal fuente de información. A nivel de país, los chilenos y colombianos reconocen ser los más fieles seguidores de programas de noticias por TV, ya que nueve de cada diez de ellos ven estos programas diariamente o algunas veces durante la semana. El segundo medio de comunicación más usado para informarse de noticias internacionales varía en cada país, pero en promedio la radio se impone con un 43 \% de audiencia, superando a periódicos e internet, que alcanzan en promedio un 34 y $33 \%$ respectivamente. Llama la atención que informarse por internet comparta el tercer lugar junto a la lectura de periódicos (ambos están dentro del intervalo de confianza de la muestra), después de la televisión o la radio. Sin embargo, esto está muy en línea con el tiempo que los latinoamericanos dedicamos para ver televisión, escuchar radio, leer periódicos o navegar por el internet, donde los dos primeros son los favoritos (LatinLink, 2015). Si bien, en promedio, la lectura de periódicos y el uso de internet para informarse son muy similares, esto es principalmente un efecto del alto consumo de diarios en el Ecuador y sobre todo en Perú, país que lideraba el ranking de lectores de periódicos en América Latina en ese tiempo (SEPP, 2015).

Al concentrarnos solo en los encuestados que manifiestan mucho a o algo de interés en los asuntos internacionales los resultados no son muy diferentes. Sin embargo, realizar un perfil de las personas que manifiestan mucho interés en los asuntos internacionales y que consumen medios de comunicación diariamente resulta muy interesante, ya que en cierta medida ellos pueden ser los líderes de opinión. 
Gráfico 3. Alto interés por noticias internacionales y consumo de medios.

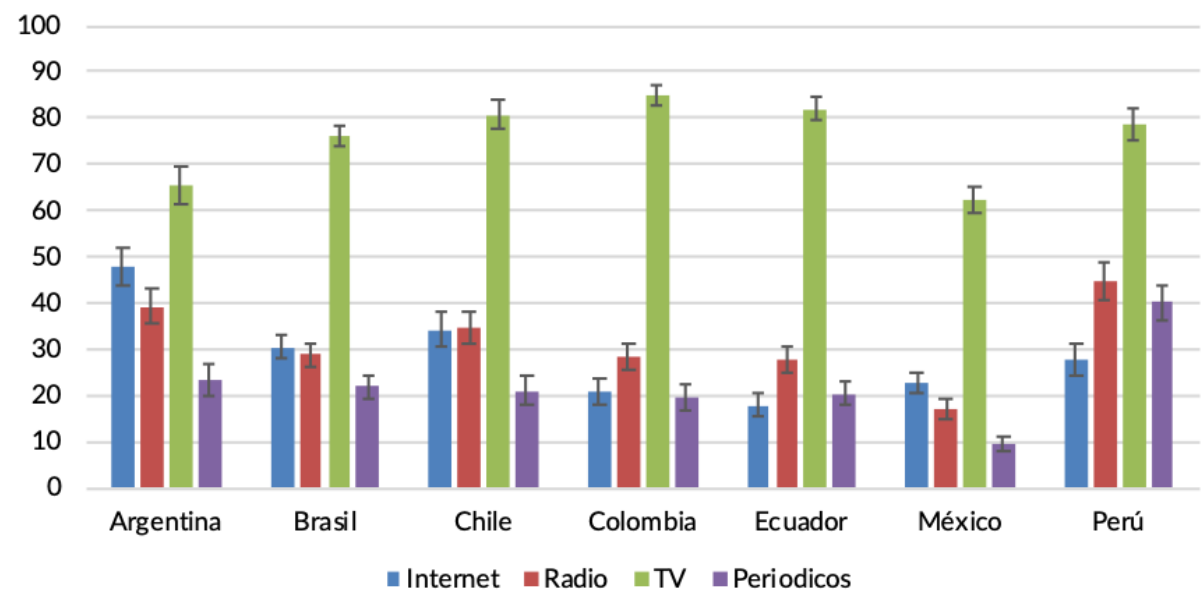

Fuente: Las Américas y el Mundo 2014.

La televisión sigue siendo el principal medio informativo para las personas que manifiestan tener mucho interés en las noticias internacionales y que usan diariamente medios de información (Gráfico 4). Si bien en promedio alcanzan $75,7 \%$, los colombianos, chilenos y ecuatorianos son los principales televidentes de noticias internacionales y los argentinos y mexicanos los más bajos. Al estudiar solamente los muy interesados en noticias extranjeras y con reconocido consumo frecuente de medios se hace interesante ver la pérdida de influencia de la radio y la potenciación del internet en muchos países. La radio sigue siendo la segunda principal fuente de información de noticias internacionales diarias para los muy interesados en el mundo en: Perú $(44,7 \%)$, Colombia $(28,4 \%)$ y Ecuador $(27,8)$. Además, la radio comparte el segundo lugar con el internet en Chile (alrededor de un $34 \%$ ), pero este último la supera con creces en Argentina (47,3\% a 39,2 \%), en Brasil (30,5 \% a $28,7 \%$ ) y en México (22,7 \% a 17,35). Los periódicos impresos son en general el medio que menos usan diariamente los más interesados en noticias internacionales, con la excepción de Perú y Ecuador, como se mencionó anteriormente. Sin embargo, esto no implica el fin de los periódicos, ya que los diarios más importantes de América Latina también se pueden leer por internet. Esta adaptación de la prensa escrita al espacio on-line ha permitido que la búsqueda de noticias nacionales o internacionales por internet sea más funcional y orientada a objetivos, como lo indica el reducido número de páginas leídas por visita (Dans, 2000, p. 4).

Una de las razones que pueden ayudar a entender por qué en un siglo dominado por la world wide web, los latinoamericanos sigan prefiriendo a la TV, se 
encuentra en las numerosas alternativas de televisión por cable y por satélite. Si bien la televisión sigue siendo la principal fuente de información sobre noticias internacionales, internet asoma como el medio del futuro, ya que la mayoría de sus usuarios son jóvenes y se está extendiendo con fuerza gracias al uso de los teléfonos inteligentes. El porcentaje de personas que usan internet en América Latina en promedio ha subido de 43,4 \% en 2015 al 71,5 \% en 2020, llegando a superar el 90 \% en países como Argentina y Chile (Internet World Stats, 2020).

\section{CONCLUSIONES}

América Latina es parte de un mundo cada vez más globalizado donde las fronteras se han hecho más difusas y la ciudadanía está cada vez más integrada en el mundo. Lo que acontece más allá de las fronteras de nuestros países nos importa muchísimo y eso se ve de manifiesto en el interés que generan las noticias internacionales, sobre todo cuando la noticia implica al país de pertenencia del individuo. Al controlar por edad no se encontraron diferencias significativas en los grados de interés, pero los resultados por sexo son sorprendentes. A diferencia de los estudios en democracias avanzadas no encontramos una brecha de género significativa en el consumo de noticias internacionales en América Latina.

Dado que este alto interés por el mundo puede ser resultado de deseabilidad social, en la encuesta también se examinó el conocimiento de asuntos internacionales. En este caso las diferencias a nivel de países se ampliaron, siendo los argentinos, colombianos y chilenos aquellos con mayores conocimientos. Sin embargo, la literatura comparada advierte que los niveles de conocimiento son altamente dependientes de la oferta informativa internacional en cada país.

Además, se ha observado que la televisión sigue siendo el principal medio de información de todo tipo y las noticias internacionales no son la excepción. En la fecha de la encuesta el internet recién estaba empezando a posicionarse como medio de información de lo internacional, pero con el avance en telefonía móvil en América Latina en los últimos años su importancia está en aumento. La importancia de los medios de comunicación en el conocimiento de lo internacional se hace más relevante si consideramos que el contacto directo a través de viajes al extranjero es relativamente bajo. La inserción internacional de la ciudadanía depende entonces de los medios de comunicación, de los avances tecnológicos y de la adecuación de los medios las nuevas tecnologías. En un mundo global donde los gobiernos nacionales están más integrados, la ciudadanía latinoamericana no se ha quedado atrás, aunque hasta ahora el entendimiento de las noticias internacionales no sea tan bueno como el interés que estas despiertan. 
MIGUEL ÁNGEL LÓPEZ VARAS

EL INTERÉS DE LOS LATINOAMERICANOS POR LOS ASUNTOS INTERNACIONALES

\section{BIBLIOGRAFÍA}

Aalberg, T., Papathanassopoulos, S., Soroka, S., Curran J., Kaori Hayashi, K., Iyengar, S., Jones, PK., Mazzoleni, G., Rojas, H., Rowe, D. y Tiffen, R. (2013). International TV News, Foreign Affairs Interest and Public Knowledge. Journalism Studies, 14(3), 387-406.

Almond, G. (1960). The American People and Foreign Policy. Praeger.

American Press Institute. (2014). Social and demographic differences in news habits and attitudes. https://www.americanpressinstitute.org/publications/reports/survey-research/ social-demographic-differences-news-habits-attitudes/

Batista, F. (2020). Assessing Political Knowledge Scales Across Countries. Evidence from Latin America. Revista Latinoamericana de Opinión Pública, 9(1), 37-62. https://doi. org/10.14201/rlop.22687

Benesch, C. (2012). An Empirical Analysis of the Gender Gap in News Consumption, Journal of Media Economics, 25(3), 147-167. https://doi.org/10.1080/08997764.2012.7 00976

Bennett, S. E., Flickinger, R. S., Baker, J. R., Rhine, S. L., y Bennett, L. L. M. (1996). Citizens' Knowledge of Foreign Affairs. Harvard International Journal of Press/Politics, 1(2), 10-29.

Caspary, W. (1970). The "Mood Theory": A Study of Public Opinion and Foreign Policy. American Political Science Review, 64(2), 536-547. https://doi.org/10.2307/1953848

Chang, T. K. (1998). All Countries Not Created Equal to Be News: World System and International Communication. Communication Research, 25(5), 528-563.

Crowne, D. P., y Marlowe, D. (1960). A new scale of social desirability independent of psychopathology. Journal of Consulting Psychology, 24(4), 349-354.

Dans, E. (2000). Internet Newspapers: Are Some More Equal than Others? Journal on Media Management, 2(1), 4-13.

Drezner, D. (2008). The Realist Tradition in American Public Opinion. Perspectives on Politics, 6(1), 51-70.

Elff, M. (2009, 2-5 abril). Political Knowledge in Comparative Perspective: The Problem of Cross- National Equivalence of Measurement [Ponencia]. MPSA Annual National Conference. Palmer House, Chicago, II.

Galtung, J., y Ruge, M. H. (1965). The structure of foreign news. Journal of Peace Research 2(1), 64-91.

Garcé, A., D’Avenia, L., López, C. y Villegas, B. (2018) Political Knowledge Regimes and policy change in Chile and Uruguay. On Think Tanks Working Paper 3.

Gilboa, E. (2002). Global Communication and Foreign Policy. Journal of Communications 52(4), 731-748.

Grasland, C. (2020). International news flow theory revisited through a space-time interaction model: Application to a sample of 320,000 international news stories published through RSS flows by 31 daily newspapers in 2015. International Communication Gazette, 82(3), 231-259.

Holsti, O. (1992). Public Opinion and Foreign Policy: Challenges to the Almond-Lippmann Consensus. Mershon Series: Research Programs and Debates. International Studies Quarterly, 36, 439-466.

Holsti, O. (2004). Public Opinion and American Foreign Policy. University of Michigan Press. Internet World Stats. (2020). https://www.internetworldstats.com/south.htm. 
Jerit, J., Barabas J and Bolsen, T. (2006). "Citizens, Knowledge, and the Information Environment." American Journal of Political Science, 50(2), 266-282.

Joye, S. (2010). Around the world in 8 pages? A longitudinal analysis of international news coverage in Flemish newspapers (1986-2006). En D. Biltereyst y S. Van Bauwel (Eds.), Working Papers Film \& TV Studies (1st ed., Vol. 2010). Academia Press.

Klingberg, F. (1952). The Historical Alternation of Moods in American Foreign Policy. World Politics, 4(2), 239-273.

LatinLink(2015)TheDataEveryLatinAmericanDigitalMarketerNeeds.http://latinlink.usmediaconsulting.com/2015/02/the-data-every-latin-american-digital-marketer-needs/

Lavrakas, P. J. (2008). Social Desirability. En Encyclopedia of survey research methods (Vols. 1-0). Sage Publications, Inc. https://dx.doi.org/10.4135/9781412963947

Lee, C. C. y Yang, J. (1996). Foreign news and national interest: Comparing U.S. and Japanese coverage of a Chinese student movement. International Communication Gazette, $56,1-18$.

Levy, D (2012) Evidence on Interest in and Consumption of Foreign News. Reuter Digital News Report. Disponible en: https://www.digitalnewsreport.org/essays/2012/ evidence-on-interest-in-and-consumption-of-foreign-news/.

Lopes, D. B. (2013). Política externa e democracia no Brasil. Editora UNESP.

Mondak, J. (2001). Developing Valid Knowledge Scales. American Journal of Political Science, 45(1), 224-238.

Morales, R y Schiavon, J. (2015). El efecto de la opinión pública en la política exterior de México: contrastando las teorías realistas y liberales. Foro internacional, 55(3), 669-706.

Morgenthau, H. (1948). Politics Among Nations. The Struggle for Power and Peace. Alfred A. Knopf.

Mouron, F., Hardt, M. S., y Onuki, J. (2018). Conocimiento político internacional a partir de un enfoque individual y de contexto: evidencia desde Brasil. Revista SAAP: Sociedad Argentina de Análisis Político, 12(1), 131-153.

Naveh, C. (2002). The Role of the Media in Foreign Policy Decision-Making: A Theoretical Framework. Conflict \& Communication online, 1(2), 1-14.

Newman, N., Dutton, W., y Blank, G. (2013). Social media in the changing ecology of news: the fourth and fifth estates in Britain. International Journal of Internet Science, 7(1), 6-22.

Norris, P. (1997). Women, media, and politics. Oxford University Press.

Nossek, H. (2004). Our News and their News: The Role of National Identity in the Coverage of Foreign News. Journalism, 5(3), 343-368.

Olmastroni, F. (2017). The alleged consensus: Italian elites and publics on foreign policy. Italian Political Science Review/Rivista Italiana Di Scienza Politica, 47(2), 149-182.

Pew Research Center. (2007). Public Knowledge of Current Affairs Little Changed by News and Information Revolutions. What Americans Know: 1989-2007. http://www.peoplepress.org/2007/04/15/public-knowledge-of-current-affairs-little-changed-by-newsand-information-revolutions/

Pew Research Center. (2018). Publics Globally Want Unbiased News Coverage, but Are Divided on Whether Their News Media Deliver. Disponible en: https://www.pewresearch.org/global/wp-content/uploads/sites/2/2018/01/Publics-Globally-WantUnbiased-News-Coverage-but-Are-Divided-on-Whether-Their-News-Media-Deliver_ Full-Report-and-Topline-UPDATED.pdf 
Pew Research Center. (2008). Where Men and Women Differ in Following the News. http://www.pewresearch.org/2008/02/06/where-men-and-women-differin-following-the-news/

Poindexter, P. M., Meraz, S., y Schmitz Weiss, A. (2008). Women, Men, and News: Divided and disconnected in the news media landscape. Routledge.

Reuters. (2014). Reuters Institute Digital News Report 2014. https://reutersinstitute.politics.ox.ac.uk/our-research/digital-news-report-2014

SEPP. (2015). Somos los mayores lectores de diarios de la región. http://sepp.pe/ somos-los-mayores-lectores-de-diarios-de-la-region/

Smith, S., Hadfield, A. and Dunne, T. (2016). Foreign Policy: Theories, Actors, Cases. Oxford University Press.

Steiner, Z. (1987). Decision-Making in American and British Foreign Policy: An Open and Shut Case. Review of International Studies, 13(1), 1-18.

$\mathrm{Wu}$, D. (2000). Systemic determinants of international news coverage: A comparison of 38 countries. Journal of Communication, 50(2), 110-130. 